\title{
Polymorphs of Tolfenamic Acids: Stability Analysis Using Cluster Method
}

\author{
Lee Sin Ang, ${ }^{1}$ Mohamed Ismail Mohamed-Ibrahim, ${ }^{2}$ and Shukri Sulaiman ${ }^{2}$ \\ ${ }^{1}$ Faculty of Applied Sciences, Universiti Teknologi MARA, 02600 Arau, Perlis, Malaysia \\ ${ }^{2}$ Computational Chemistry and Physics Laboratory, School of Distance Education, Universiti Sains Malaysia, \\ 11800 Pulau Pinang, Malaysia
}

Correspondence should be addressed to Lee Sin Ang; anglee631@perlis.uitm.edu.my

Received 2 October 2015; Revised 2 March 2016; Accepted 14 March 2016

Academic Editor: Ravindra R. Pandey

Copyright (c) 2016 Lee Sin Ang et al. This is an open access article distributed under the Creative Commons Attribution License, which permits unrestricted use, distribution, and reproduction in any medium, provided the original work is properly cited.

We report results of the relative stability between form I and form II of tolfenamic acid. By performing systematic cluster calculations at the $\mathrm{B} 3 \mathrm{LYP} / 6-31 \mathrm{G}^{*}$ level of theory and including the corrections to the dispersion and basis set superposition error, we found that form II is energetically more stable than form I. Furthermore, we found that the formation of dimers has a stabilizing effect compared to individual monomers in the clusters that we have considered.

\section{Introduction}

Polymorphs are compounds with the same chemical formula but with different arrangements of the molecules in the solid state. Due to the difference in the physical characteristics between the polymorphs, an understanding of the polymorphs is of the utmost importance for the proper usage of a compound. The studies of polymorphs involve diverse fields, employing experimental and theoretical approaches. However, even though studies are aplenty in this field, there are still questions to be answered, as pointed out in a few recent review articles on polymorphism [1,2]. To elucidate the stability between different polymorphs, the concept of relative energy is usually used. Apart from the molecular energy or the lattice energy, the energy of molecular conformers and the crystal lattice energies have also been put forward $[3,4]$. Since there are interactions between molecules in the crystal, by including the lattice energy, this approach takes into consideration the interaction energy between the molecules and avoids assessing the stability of the polymorphs based on just the stability of the molecular conformer.

In this report, the focus is on a compound known as tolfenamic acid, a nonsteroidal anti-inflammatory drug. Tolfenamic acid was initially found in two forms, the white (form I) and the yellow (form II) [5]. A decade later, this compound was reported to exist in five polymorphic forms [6]. The two most studied forms are shown in Figure 1. The comparison of the stability of tolfenamic acid was first given by Andersen et al., where it was reported that the yellow form is more stable than the white form based on their analysis of the crystal structures and their physical properties [5]. This is in agreement with the results from the theoretical consideration of the total energy, studied using the periodic calculations [7]. The same conclusion was obtained by Mattei and Li from the calculations of molecules in the gas phase $[8,9]$. In addition, these results are in agreement when the density of the forms is considered, where the yellow form is denser than the white form [7]. However, disagreement arises when comparison between the polymorphs is made using the lattice energy. Using solution calorimetry, Surov et al. found that the yellow form is more stable than the white form by $6.7 \pm 1.2 \mathrm{~kJ} \mathrm{~mol}^{-1}$ [7]. This result contradicted with the ones reported by Mattei and $\mathrm{Li} \mathrm{[9]} \mathrm{and} \mathrm{Uzoh} \mathrm{et} \mathrm{al.} \mathrm{[10],} \mathrm{where} \mathrm{the} \mathrm{white} \mathrm{form} \mathrm{is} \mathrm{more}$ stable than the yellow form by $\sim 3 \mathrm{~kJ} \mathrm{~mol}^{-1}$ and $2 \mathrm{~kJ} \mathrm{~mol}^{-1}$, respectively.

In this study, we embarked on the objective to clarify the contradicting trend of the stability of form I and form II of tolfenamic acid. We will provide energetic perspective from the cluster method. The cluster method is different from other methods as it incorporates the molecules in an explicit 


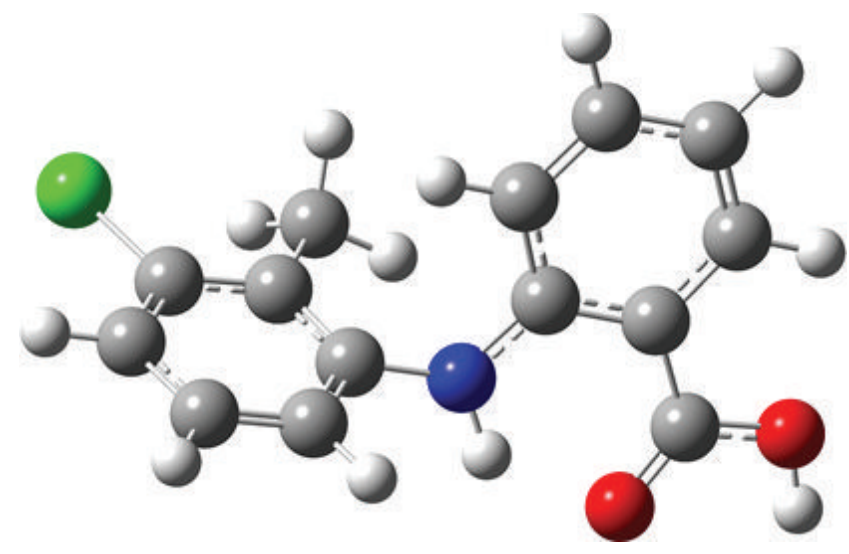

(a)

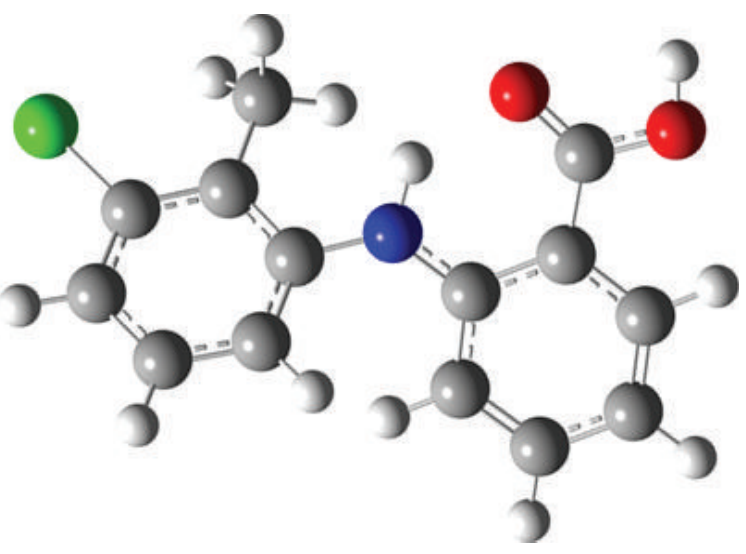

(b)

FIGURE 1: Molecules of tolfenamic acid in polymorphic forms. (a) Form I and (b) form II. Chlorine is represented in green, carbon grey, nitrogen blue, oxygen red, and hydrogen white. The diagrams were generated using Gauss View [16] using the X-ray data from [5].

way such that the total energy can include the intermolecular interaction energy. Provided that the size of the cluster is sufficiently large, the interaction energies between the molecules will be mimicking the lattice energy. This method corrects the deficiency of certain conformational analysis which only considers the molecule in the gas phase and hence the neglect of intermolecular energies and the crystal packing effect. The details of the method will be discussed in the next section.

\section{Methodology}

We performed systematic cluster calculations on the energetic variations when the number of molecules was increased. The coordinates of form I and form II molecules were obtained from the Crystallography Information File associated with the paper by Andersen et al. [5]. To generate the surrounding molecules of a central molecule, we used the "Molecular Shell" feature in the Mercury program [11]. By first selecting the nitrogen atom of the central molecule, we generated its neighbor molecules by specifying the "contact distance range" values, and the molecules that are within that particular contact distance will be shown. By specifying larger values, more molecules will be included. This should give better representation of the intermolecular energy and hence mimic the lattice energy. Starting from $4 \AA$, we increased the contact distance in steps of $1 \AA$ until $10 \AA$. By doing this, we have nine clusters (including the one-molecule and dimer cluster) for each of forms I and II. However, some of the clusters generated from the Mercury program contain molecules which are incomplete dimers. Therefore, extra molecules were added manually to complete the incomplete dimers. Hence, we have another set of clusters with complete dimers. With this, we can compare the results between clusters that contained molecules that are paired and unpaired as dimers. The largest cluster in this study consists of 36 molecules, which translates into 1080 atoms. It is generated in form II, with contact distance of $8 \AA$, and extra molecules were added so as to form dimers. Figure 2 shows two of the manymolecule clusters modeled.

Computations were performed using G09 suite of programs utilizing DFT at B3LYP level of theory with $6-31 \mathrm{G}^{*}$ as the basis set [12]. Corrections to the basis set superposition error (BSSE) were performed using the geometrical counterpoise program (gCP) [13]. As for the improvement of the B3LYP method to model the dispersion energy, the scheme known as D3 by Grimme et al. [14, 15] was used.

The relative energy per molecule $\left(E_{\text {rel }}\right)$ was used to indicate the relative stability. The energies of the molecular crystal were obtained by using a fragment of the crystal. This quantity has the form similar to that used to find the lattice energy:

$$
\begin{gathered}
E_{\text {rel }}=\frac{E_{\text {total }}}{n}-E_{\text {molecule }}, \\
E_{\text {total }}=E_{\mathrm{scf}}+E_{\mathrm{D} 3}+E_{\mathrm{gCP}},
\end{gathered}
$$

where $E_{\text {total }}$ is the total energy of the cluster (including the corrective energies of dispersion $\left(E_{\mathrm{D} 3}\right)$ and $\left.\mathrm{gCP}\left(E_{\mathrm{gCP}}\right)\right), n$ is the number of molecules in the cluster, $E_{\text {molecule }}$ is the energy of the single molecule, and $E_{\text {scf }}$ is the SCF energy from G09.

\section{Results and Discussion}

The results for the systematic cluster calculations are tabulated in Tables 1 and 2 and displayed graphically in Figure 3. It has to be pointed out that the inclusion of the gCP and D3 corrections (not shown in the tables) rendered the $E_{\text {total }} / n$ more negative than those without (more negative $E_{\text {total }} / n$ is more stable). As D3 are negative values, while gCP are positive, the net result is the increased cohesiveness of the molecules. Hence, the inclusion of the correction schemes gave a more strongly bounded picture for the molecules.

The stability of the structures can be seen from the energy per molecule $\left(E_{\text {total }} / n\right)$. By considering $E_{\text {total }} / n$ of the clusters with completing dimers (the third column in Tables $1(\mathrm{~b})$ and 


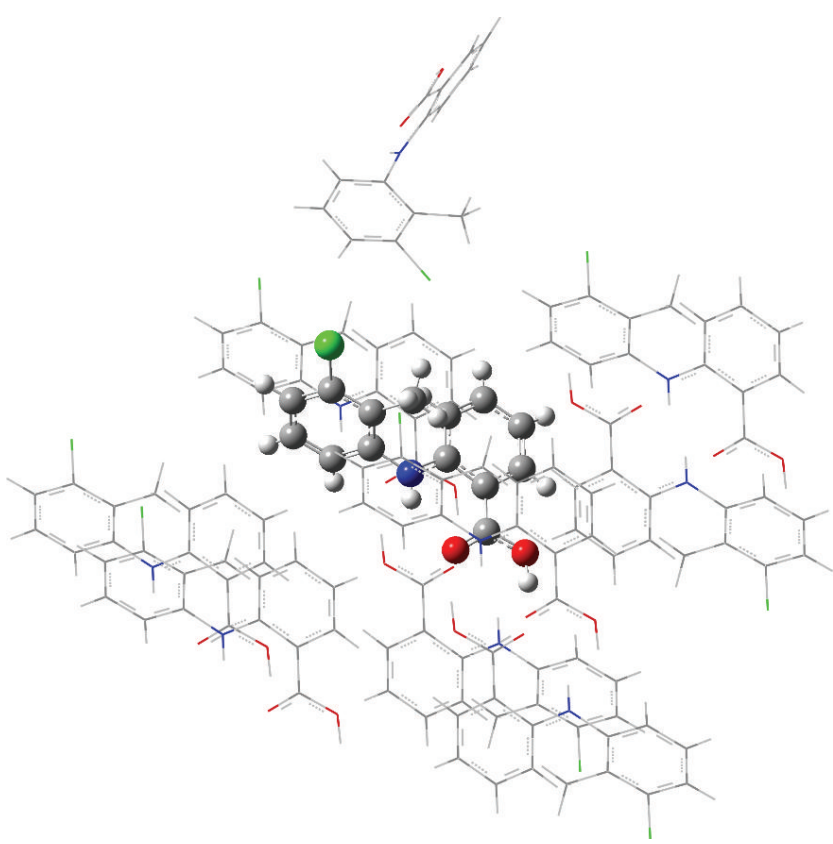

(a)

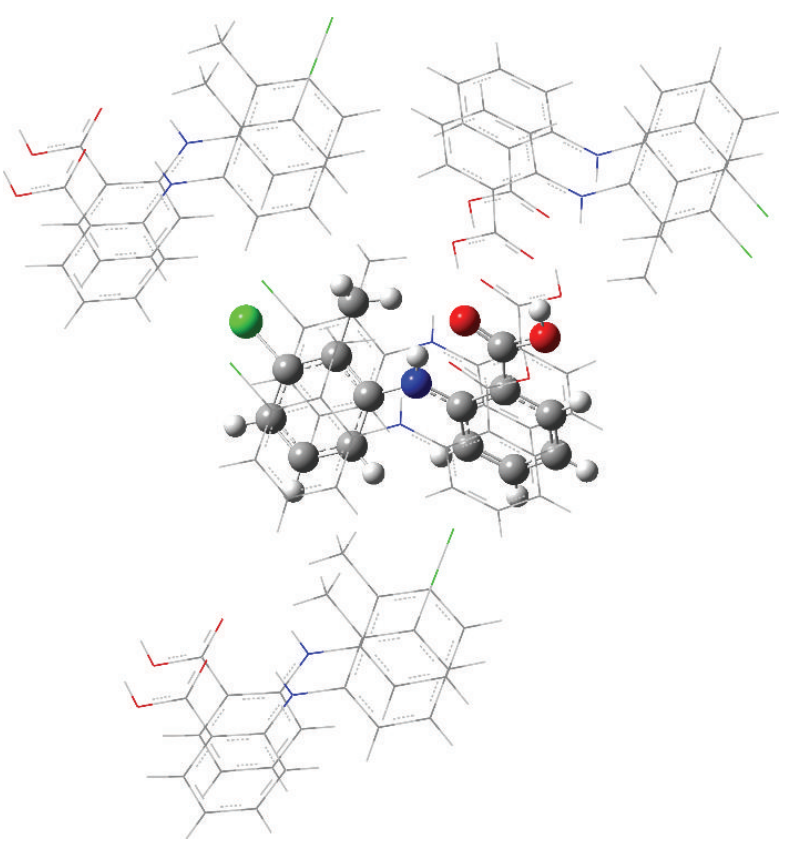

(b)

FIGURE 2: Many-molecule cluster of forms I and II. (a) Cluster of form I with 10 molecules and (b) cluster of form II with 9 molecules. For clarity, the center molecules are displayed with ball-and-stick form, while the neighboring molecules are in wireframe form.

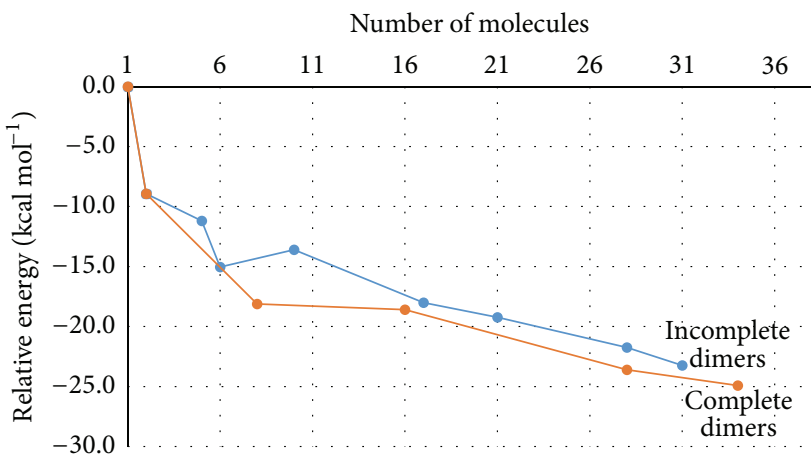

(a) Relative energy versus number of molecules: form I

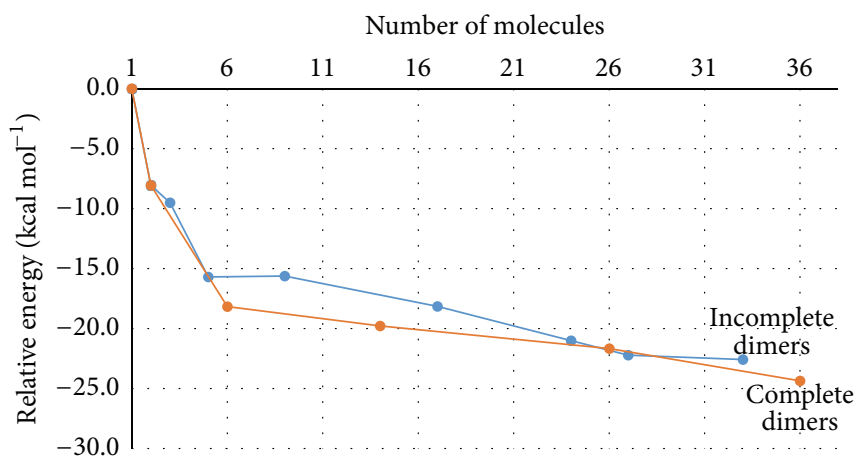

(b) Relative energy versus number of molecules: form II

FIGURE 3: Changes of $E_{\text {rel }}$ as the clusters become larger for (a) form I and (b) form II. The lines are guidance to the changes in $E_{\text {rel }}$.

2(b)), form I has $E_{\text {total }} / n$ that is always more positive than the one in form II. This showed that form II is more stable than form I. This result agrees with the ones reported by Surov et al. using the periodic code [7] and Mattei and Li's gas phase calculations $[8,9]$. We anticipate that, with the inclusion of more molecules in a cluster, $E_{\text {total }} / n$ will eventually reach a stable limit.

We can see that, in Tables 1 and 2 and Figure 3, as the number of molecules is increased, the relative energy of the molecules, $E_{\text {rel }}$, in larger clusters is decreasing (becoming more negative). This is in line with the notion that as the cluster becomes larger, there are more interactions between the molecules. From Tables 1 and 2, we found that as extra molecules were added to the existing clusters to create dimers in the clusters, the relative energies are generally lower. Without the completing molecules, there were fluctuations of $E_{\text {rel }}$, with some $E_{\text {rel }}$ in larger clusters having a more positive value than those of smaller cluster (as seen in the blue curves of Figures 3(a) and 3(b)), indicating that some of the molecules in smaller cluster are more stable than those in the larger clusters. This result may be due to the stabilizing effect of the intermolecular interactions between the two molecules that form the dimer. The existence of the molecules as dimers, as in the solid state, has strengthened the overall interactions in the cluster. This stabilizing effect is absent for incomplete dimers. Thus, models with incomplete dimers will 
TABLE 1: Energies of form I clusters. (a) Without complete dimers and (b) with complete dimers. ${ }^{*}$ denotes cluster with complete dimers.

(a)

\begin{tabular}{lccc}
\hline Molecule in cluster, $n$ & $E_{\text {total }} / n(\mathrm{au})$ & $E_{\text {rel }}(\mathrm{au})$ & $E_{\text {rel }}\left(\mathrm{kcal} \mathrm{mol}^{-1}\right)$ \\
\hline 1 & -1205.8408931 & 0.0000000 & 0.0000000 \\
2 & -1205.8551323 & -0.0142392 & -8.9351323 \\
5 & -1205.8587378 & -0.0178447 & -11.1975784 \\
6 & -1205.8648680 & -0.0239749 & -15.0442962 \\
10 & -1205.8625549 & -0.0216618 & -13.5928331 \\
17 & -1205.8695943 & -0.0287012 & -18.0101120 \\
21 & -1205.8715498 & -0.0306567 & -19.2371569 \\
28 & -1205.8755554 & -0.0346623 & -21.7507209 \\
31 & -1205.8779238 & -0.0370307 & -23.2369032 \\
\hline
\end{tabular}

(b)

\begin{tabular}{lccc}
\hline Molecule in cluster, $n$ & $E_{\text {total }} / n(\mathrm{au})$ & $E_{\text {rel }}(\mathrm{au})$ & $E_{\text {rel }}\left(\mathrm{kcal} \mathrm{mol}^{-1}\right)$ \\
\hline 1 & -1205.8408931 & 0.0000000 & 0.0000000 \\
2 & -1205.8551323 & -0.0142392 & -8.9351323 \\
$8^{*}$ & -1205.8697762 & -0.0288831 & -18.1242362 \\
$16^{*}$ & -1205.8705116 & -0.0296185 & -18.5857075 \\
$28^{*}$ & -1205.8785210 & -0.0376279 & -23.6116018 \\
$34^{*}$ & -1205.8806005 & -0.0397074 & -24.9165293 \\
\hline
\end{tabular}

TABle 2: Form II. (a) Without complete dimers and (b) with complete dimers. ${ }^{*}$ denotes cluster with complete dimers.

(a)

\begin{tabular}{lcccc}
\hline Molecule in cluster, $n$ & $E_{\text {total }}(\mathrm{au})$ & $E_{\text {total }} / n(\mathrm{au})$ & $E_{\text {rel }}(\mathrm{au})$ & $E_{\text {rel }}\left(\mathrm{kcal} \mathrm{mol}^{-1}\right)$ \\
\hline 1 & -1205.9611637 & -1205.9611637 & 0.0000000 & 0.0000000 \\
2 & -2411.9480238 & -1205.9740119 & -0.0128482 & -8.0623068 \\
3 & -3617.9289916 & -1205.9763305 & -0.0151668 & -9.5172409 \\
5 & -6029.9308966 & -1205.9861793 & -0.0250156 & -15.6973945 \\
9 & -10853.8745303 & -1205.9860589 & -0.0248952 & -15.6218424 \\
17 & -20501.8317720 & -1205.9901042 & -0.0289406 & -18.1602843 \\
24 & -28943.8715796 & -1205.9946492 & -0.0334855 & -21.0122328 \\
27 & -32561.9074876 & -1205.9965736 & -0.0354099 & -22.2198412 \\
33 & -39797.9062256 & -1205.9971584 & -0.0359947 & -22.5867651 \\
\hline
\end{tabular}

(b)

\begin{tabular}{lcccc}
\hline Molecule in cluster, $n$ & $E_{\text {total }}(\mathrm{au})$ & $E_{\text {total }} / n(\mathrm{au})$ & $E_{\text {rel }}(\mathrm{au})$ & $E_{\text {rel }}\left(\mathrm{kcal} \mathrm{mol}^{-1}\right)$ \\
\hline 1 & -1205.9611637 & -1205.9611637 & 0.0000000 & 0.0000000 \\
2 & -2411.9480238 & -1205.9740119 & -0.0128482 & -8.0623068 \\
$6^{*}$ & -7235.9407396 & -1205.9901233 & -0.0289596 & -18.1722298 \\
$14^{*}$ & -16883.8977160 & -1205.9926940 & -0.0315303 & -19.7853708 \\
$26^{*}$ & -31355.8884529 & -1205.9957097 & -0.0345460 & -21.6777488 \\
$36^{*}$ & -43415.9991369 & -1205.9999760 & -0.0388123 & -24.3548628 \\
\hline
\end{tabular}

give the incorrect pictures of the stability of the clusters. This is especially true when the number of molecules in the cluster is low.

As the size of the clusters increases, generally, the decrease of the relative energy is smaller. The trend can be seen from the last points of the graphs in Figures 3(a) and 3(b), where the gradient of the curve is decreasing compared to the initial increase from the 1-molecule cluster. However, calculations involving larger clusters are out of reach of our current computing capability.

\section{Conclusion}

We have performed calculations involving forms I and II of tolfenamic acid. By systematic calculations using B3LYP/6$31 G^{*}$ with correctional schemes, it was found that form II is 
more stable than form I. We also found that, for the molecules of forms I and II of tolfenamic acid, the relative energies were influenced by the formation of complete dimers. For clusters with complete dimers, $E_{\text {rel }}$ is lower compared to clusters of similar size but with incomplete dimers. We attribute this to the fact that intermolecular interactions between molecules in a complete dimer are energetically more favorable compared to a molecule in an incomplete dimer.

\section{Competing Interests}

The authors declare that they have no competing interests.

\section{References}

[1] A. J. Cruz-Cabeza and J. Bernstein, "Conformational polymorphism," Chemical Reviews, vol. 114, no. 4, pp. 2170-2191, 2014.

[2] D. Braga, F. Grepioni, L. Maini, and M. Polito, "Crystal polymorphism and multiple crystal forms," Structure and Bonding, vol. 132, pp. 25-50, 2009.

[3] A. Nangia, "Conformational polymorphism in organic crystals," Accounts of Chemical Research, vol. 41, no. 5, pp. 595-604, 2008.

[4] A. Nangia, "Molecular conformation and crystal lattice energy factors in conformational polymorphs," in Models, Mysteries and Magic of Molecules, J. A. Boeyens and J. F. Ogilvie, Eds., p. 63, Springer, Amsterdam, Netherlands, 2008.

[5] K. V. Andersen, S. Larsen, B. Alhede, N. Gelting, and O. Buchardt, "Characterization of two polymorphic forms of tolfenamic acid, $N$-(2-methyl-3-chlorophenyl)anthranilic acid: their crystal structures and relative stabilities," Journal of the Chemical Society, Perkin Transactions, vol. 2, no. 10, pp. 14431447, 1989.

[6] V. López-Mejías, J. W. Kampf, and A. J. Matzger, "Polymerinduced heteronucleation of tolfenamic acid: structural investigation of a pentamorph," Journal of the American Chemical Society, vol. 131, no. 13, pp. 4554-4555, 2009.

[7] A. O. Surov, P. Szterner, W. Zielenkiewicz, and G. L. Perlovich, "Thermodynamic and structural study of tolfenamic acid polymorphs," Journal of Pharmaceutical and Biomedical Analysis, vol. 50, no. 5, pp. 831-840, 2009.

[8] A. Mattei and T. Li, "Interplay between molecular conformation and intermolecular interactions in conformational polymorphism: a molecular perspective from electronic calculations of tolfenamic acid," International Journal of Pharmaceutics, vol. 418, no. 2, pp. 179-186, 2011.

[9] A. Mattei and T. Li, "Polymorph formation and nucleation mechanism of tolfenamic acid in solution: an investigation of pre-nucleation solute association," Pharmaceutical Research, vol. 29, no. 2, pp. 460-470, 2012.

[10] O. G. Uzoh, A. J. Cruz-Cabeza, and S. L. Price, "Is the fenamate group a polymorphophore? Contrasting the crystal energy landscapes of fenamic and tolfenamic acids," Crystal Growth \& Design, vol. 12, no. 8, pp. 4230-4239, 2012.

[11] C. F. Macrae, I. J. Bruno, J. A. Chisholm et al., "Mercury CSD 2.0- new features for the visualization and investigation of crystal structures," Journal of Applied Crystallography, vol. 41, no. 2, pp. 466-470, 2008.

[12] M. J. Frisch, G. W. Trucks, H. B. Schlegel et al., Gaussian 09, Revision A.01, Gaussian, Wallingford, Conn, USA, 2009.
[13] H. Kruse and S. Grimme, "A geometrical correction for the inter- and intra-molecular basis set superposition error in Hartree-Fock and density functional theory calculations for large systems," The Journal of Chemical Physics, vol. 136, no. 15, Article ID 154101, 2012.

[14] S. Grimme, J. Antony, S. Ehrlich, and H. Krieg, "A consistent and accurate $a b$ initio parametrization of density functional dispersion correction (DFT-D) for the 94 elements $\mathrm{H}-\mathrm{Pu}$," The Journal of Chemical Physics, vol. 132, no. 15, Article ID 154104, 2010.

[15] S. Grimme, S. Ehrlich, and L. Goerigk, "Effect of the damping function in dispersion corrected density functional theory," Journal of Computational Chemistry, vol. 32, no. 7, pp. 14561465, 2011.

[16] R. Dennington, T. Keith, and J. Millam, GaussView 5, Semichem, Shawnee Mission, Kan, USA, 2009. 

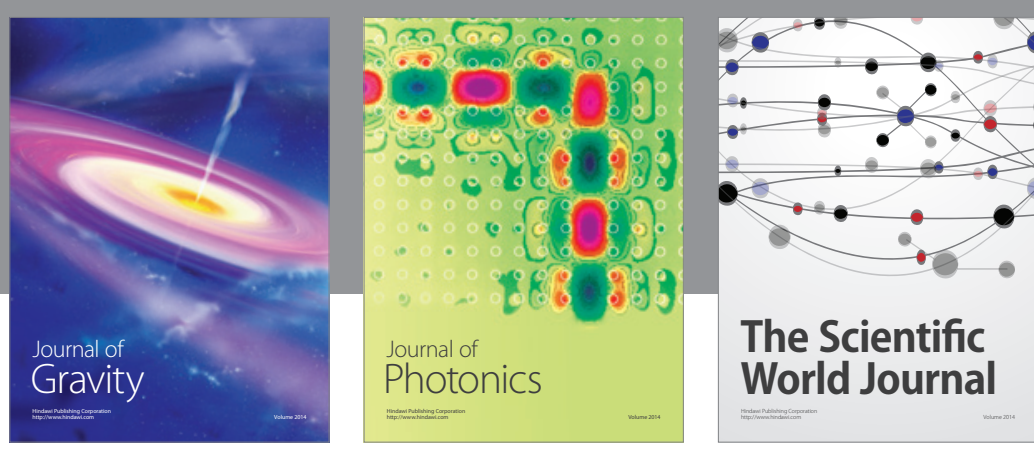

The Scientific World Journal
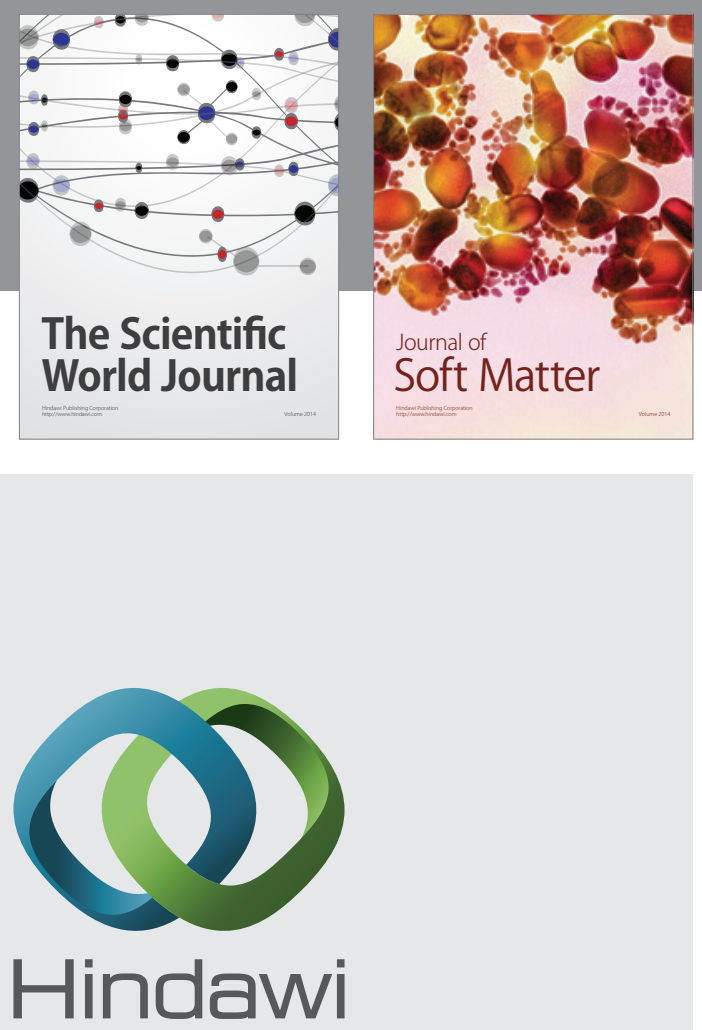

Submit your manuscripts at

http://www.hindawi.com

nternational Journal of

Statistical Mechanics
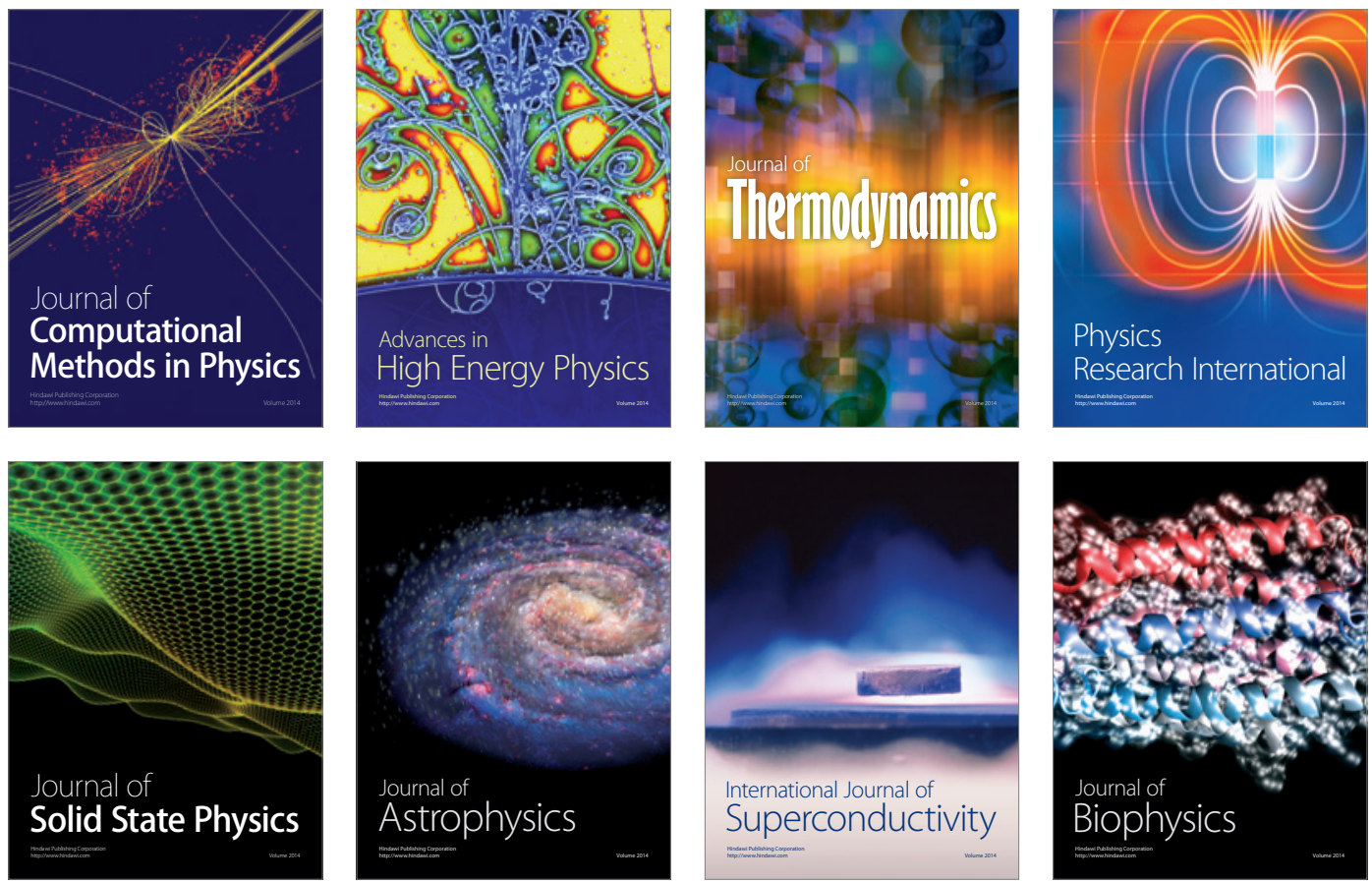
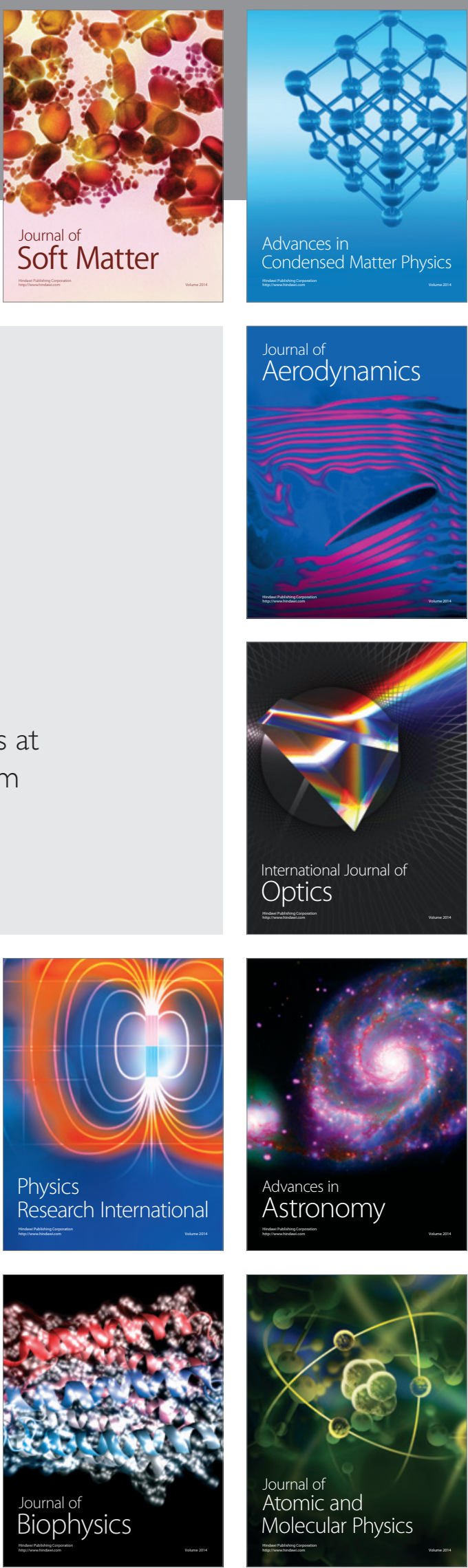